\title{
Effects of d-electrons in pseudopotential screened-exchange density functional calculations
}

\author{
Byounghak Lee, ${ }^{*}$ Andrew Canning, and Lin-Wang Wang \\ Computational Research Division, Lawrence Berkeley \\ National Laboratory, Berkeley, California 94720
}

(Received October 2, 2006)

\begin{abstract}
We report a theoretical study on the role of shallow $d$ states in the screened-exchange local density approximation (sX-LDA) band structure of binary semiconductor systems. We found that the inaccurate pseudo-wavefunctions can lead to 1) an overestimation of the screened-exchange interaction between the localized $d$ states and the delocalized higher energy $s$ and $p$ states and 2) an underestimation of the screened-exchange interaction between the $d$ states. The resulting sX-LDA band structures have substantially smaller band gaps compared with experiments. We correct the pseudo-wavefunctions of $d$ states by including the semicore $s$ and $p$ states of the same shell in the valence states. The correction of pseudo-wavefunctions yields band gaps and the $d$ state binding energy with good agreements with experiments and the full potential linearized augmented planewave (FLAPW) calculations. Compared with the quasi-particle GW method, our sX-LDA results shows not only similar quality on the band gaps but also much better $d$ state binding energy. Combined with its capability of ground state structure calculation, the sX-LDA is expected to be a valuable theoretical tool for the II-VI and III-V (especially the III-N) bulk semiconductors and nanostructure studies.
\end{abstract}

PACS numbers: 71.15.Mb, 71.20.Nr

\footnotetext{
*Electronic address: bhlee@lbl.gov
} 


\section{INTRODUCTION}

The vast majority of modern ab initio condensed matter calculations are based on the density functional theory (DFT). [1] The Kohn-Sham (KS) scheme[2] made it possible to apply the DFT to realistic systems by mapping the many-body systems to auxiliary single particle systems, and has been proven to be extremely successful for the ground state properties. Among different numerical methods to solve the KS equations, the pseudopotential (PP) methods are particularly favored due to its computational efficiency resulting from the replacement of the sharp ionic potential with a much softer one.[3-5] The remarkable success of the PP method relies on the fact that the core states, i.e., the states whose atomic eigenvalues are much lower than the valence states, are inert to the changes in the electron density outside of the so called core-radius. The norm conservation condition on the PP's guarantees that the net electron density in PP calculation inside the core-radius agree with the electron density in the all-electron (AE) calculations and, at the same time, the wavefunctions outside of the core-radius are the same in PP and AE methods.

The screened-exchange local density approximation (sX-LDA) method was proposed as an example of the generalized Kohn-Sham (GKS) scheme as a means of overcoming the wellknown band gap problem in KS schemes. [6, 7] While still within the auxiliary non-interacting electron scheme, GKS includes the energy functional which has an explicit wavefunction dependence. It has been demonstrated that the sX-LDA within the plane-wave pseudopotential method are very successful for many of group III-V and IV semiconductors.[7] Preliminary calculations on group II-VI and some of group III-V semiconductors, however, show that sX-LDA method with LDA norm conserving PPs yields poor band gaps. The common features of these semiconductors is the presence of shallow cation $d$ states. In contrast, it has been reported that the sX-LDA without PP's within full-potential linearized augmented planewave (FLAPW) method is accurate regardless of the presence of shallow $d$ states.[8] In this paper, we discuss the role of the shallow $d$ states in SX-LDA method.

Our major findings are as follow. 1) In the presence of shallow $d$ states, using the conventional LDA pseudopotentials the sX-LDA predicts band gaps are substantially smaller than experiments. This is due to the pseudo-wavefunction error in the LDA pseuodopotentials. 2) The sX-LDA band gap can be corrected by including the semicore $s$ and $p$ states. This procedure improves the nonlocal screened-exchange interaction by forcing the valence 
pseudowavefunctions to agree with all-electron wavefunction outside a very small radius. 3) The inclusion of semi-core states also improves the $d$ state position in sX-LDA. While the sX-LDA band gap is comparable to the GW band gap, the $d$ state position in sX-LDA is actually in much better agreement with experiments.

\section{MEHTODS}

The single particle states in sX-LDA method are found by minimizing the total energy defined as

$$
E_{\mathrm{tot}}[v]=T+E_{\mathrm{H}}[\rho]+E_{\mathrm{sX}}[\{\psi\}]+R[\rho]+E_{\text {ext }}[v]
$$

with the electron density defined as

$$
\rho(\mathbf{r})=\sum_{i}^{o c c}\left|\psi_{i}(\mathbf{r})\right|^{2}
$$

In Eq.(1), $T, E_{\mathrm{H}}[\rho]$, and $E_{\text {ext }}[v]$ are the kinetic energy, the direct Hartree Coulomb energy, and the external potential energy, respectively. The screened-exchange energy is defined with a Thomas-Fermi screening function;

$$
E_{\mathrm{sX}}[\{\psi\}]=-\frac{1}{2} \sum_{i, j}^{\mathrm{occ}} \iint d \mathbf{r} d \mathbf{r}^{\prime} \frac{\psi_{i}^{*}(\mathbf{r}) \psi_{j}^{*}\left(\mathbf{r}^{\prime}\right) \psi_{j}(\mathbf{r}) \psi_{i}\left(\mathbf{r}^{\prime}\right) e^{-k_{T F}\left|\mathbf{r}-\mathbf{r}^{\prime}\right|}}{\left|\mathbf{r}-\mathbf{r}^{\prime}\right|}
$$

where $k_{T F}$ is the screening length determined from the average density of electrons. $R[\rho]$ is the difference between the LDA exchange-correlation energy and the local approximation of the screened-exchange energy, $E_{\mathrm{sx}}[\{\psi\}]$;

$$
R[\rho]=E_{\mathrm{xc}}^{\mathrm{LDA}}[\rho]-E_{\mathrm{sX}}^{\mathrm{loc}}[\rho]
$$

Note that PP's are usually derived from the LDA formalism and, as a result, they do not guarantee the adequacy in the nonlocal sX-LDA formalism. Strictly speaking, this way of constructing PP's is not consistent with the sX-LDA approach. A better way is to contruct a PP with its sX-LDA pseudopotential and pseudo wavefunction results agree with the all electron results (e.g, construct the PP within the sX-LDA formalism). It is known from the exact exchange (EXX) method that PP's generated within EXX can be substantially different from the one generated within LDA.[9] It has also been studied that the optimal screening length of sX-LDA method in the atomic configurations is very different from the 
bulk Thomas-Fermi screening length.[10] However, because of the nonlocality of the sXLDA formalism, just as in Harree-Fock theory [? ], there is no rigorous way to generate the sX-LDA pseudopotentials. To our knowledge, all the previous planewave sX-LDA studies have used PP's generated within the LDA method. This situation is rather analogous to that of GW calculations, where the LDA PP's are used without any modification. The most desirable approach is to include all the electrons, just like what is implemented in the FLAPW method. But to use planewave as the basis to calculate larger systems, one has to use PP. This is the topic of the current paper. Our previous studies indicate that the LDA PP's work well for sX-LDA when $d$ states are not present. Therefore, we believe it is a practical approach to apply the LDA PP's to the sX-LDA formalism and to understand any problem in the LDA-PP sX-LDA calcualtions, and to correct these problems based on our understanding.

The question regarding the LDA PP's in sX-LDA arises from the orbital dependence of $E_{\mathrm{sX}}[\{\psi\}]$. The difference between the all-electron wavefunctions and the pseudowavefunction in the core region causes errors in the screened-exchange integral of $\mathrm{Eq}(3)$, which has not been taken into account in the PP generation based on LDA formalism. To investigate the effects of PP in the presence of shallow $d$-states, we follow the procedure prescribed in GW studies by Rohlfing et al.[11] and Luo et al..[12] Two sets of cation atom PP's are studied; 1$)(+12 /+13)$ PP's with $d$ electrons and the outer shell electrons in the valence. The numbers in the parenthesis denotes the number of valence electrons used in the PP calculation for column II and III cations respectively. 2) $(+20 /+21)$ PP's with entire semicore $s, p$, and $d$ electrons as well as the outer shell electrons in the valence. For example, in case of $\mathrm{Zn}$ atom, (+12) Zn PP includes $3 d$ and $4 s$ electrons in the valence. (+20) Zn PP, on the other hand, includes $3 s, 3 p, 3 d$, and $4 s$ electrons in the valence. For lower charge state cation atoms, i.e. $(+12 /+13) \mathrm{PP}$, we use the conventional norm conversing PP's. For higher charge state cation atoms, i.e. $(+20 /+21)$ PP, we first construct the PP for an ion without the outer shell electrons and then vary the core-radius of each PP angular momentum channel to fit the eigenvalues of the neutral atom configuration. For instance, for a $\mathrm{Zn}$ atom we first build (Ne) $3 s^{2} 3 p^{6} 3 d^{10}$ PP's. This $(+20)$ Zn PP is used for (Ne) $3 s^{2} 3 p^{6} 3 d^{10} 4 s^{2}$ configuration, and the eigenvalues are compared with the AE calculation results. This complicated procedure is required because, in the conventional pseudopotential generators, only one reference state for one angular momentum can be used. Our PP's yield good outer 
shell eigenvalues with the energy discrepancy $<0.1 \mathrm{eV}$ and the $d$ state eigenvalues with the energy discrepancy $\lesssim 0.15 \mathrm{eV}$. This is shown in Table I. Although there is a small difference in the $d$ state energy, we found that this does not cause any significant change in the band gap in following calculations.

The effects of inclusion of semicore states on the valence pseudo-wavefunctions are shown in Fig. 1. The most notable difference between $(+12) \mathrm{Zn} \mathrm{PP}$ wavefunctions and (+20) Zn $\mathrm{PP}$ is the overall location of the $d$ orbitals. The center of mass of the all electron $d$ orbital and that of $(+20) \mathrm{Zn} \mathrm{PP}$ is much closer to the nucleus than the $d$ orbital of $(+12) \mathrm{Zn} \mathrm{PP}$. This results in the much smaller overlap between the $d$ states and the delocalized $s$ and $p$ states. In addition, the nodal structure of the valence wavefunctions are changed thanks to the orthonormality condition with the semicore states.

\section{RESULTS AND DISCUSSIONS}

The error introduced by the usage of the pseudopotentials can be analyzed in terms of the screened-exchange integral between the atomic orbitals. The screened-exchange interaction between the atomic orbitals $\left\{\phi_{\alpha}\right\}$ is

$$
K_{\alpha \beta \gamma \delta}=\int d \mathbf{r}_{1} \int d \mathbf{r}_{2} \phi_{\alpha}^{*}\left(\mathbf{r}_{1}\right) \phi_{\beta}^{*}\left(\mathbf{r}_{2}\right) \phi_{\gamma}\left(\mathbf{r}_{1}\right) \phi_{\delta}\left(\mathbf{r}_{2}\right) \frac{e^{-k_{T F}\left|\mathbf{r}_{1}-\mathbf{r}_{2}\right|}}{\left|\mathbf{r}_{1}-\mathbf{r}_{2}\right|}
$$

where $\alpha, \beta, \gamma$, and $\delta$ are the composite quantum numbers consist of the orbital angular momenta, $l$ and $m$, and the spin, $\sigma$. Note that the opposite spins do not contribute to the exchange integral. As for the screening length, $k_{T F}$, we have chosen a typical bulk screening length using the average valence charge density, including $s$ and $p$ states. In Table I we show the screened-exchange integral $K_{l, l^{\prime}} \equiv K_{l, l^{\prime}, l^{\prime}, l}$ of neutral atomic configurations. For the sake of simplicity, we averaged the integral over the magnetic quantum number, $m$, and restricted ourselves to the integral of the two orbitals $l$ and $l^{\prime}$. The screened-exchange integral between the highest $s$ and $p$ states, i.e., $K_{s s}, K_{p p}$, and $K_{s p}$, are almost identical to AE results regardless of the PP's. This is because the $s$ and $p$ wavefunctions have nodes at similar radius and, as a result, they have little weight in the nodal points. Therefore, the nodal structure of these wavefunctions are of less importance in the exchange integral between themselves. In the case of $d$ orbitals, however, the nodal structure plays important role in the screened-exchange, conspiring with the center of mass position of the wavefunctions. The 
larger overlap between the $d$ orbitals and the $s$ and $p$ orbitals of $(+12 /+13) \mathrm{PP}$ is reflected in the large $K_{s d}(+12 /+13) / K_{s d}(A E)$ and $K_{p d}(+12 /+13) / K_{p d}(A E)$. The larger error of $K_{s d}(+20 /+21)$ and $K_{p d}(+20 /+21)$ relative to $K_{d d}(+20 /+21)$ probably stems from the nodal structure error in the $s$ and $p$ pseudo-wavefunctions. The screened exchange is smaller for wider spread wavefunctions because of the $e^{-k_{T F} r} / r$ screened Coulomb interaction. $d-d$ overlap, therefore, is much smaller in $(+12 /+13) \mathrm{PP}$ calculations than in $(+20+21) \mathrm{PP}$ and the all-electron calculations.

Having established the fact that the full semicore states are necessary for proper $d$ orbitals in sX-LDA, we calculate the electronic structure of test systems where the shallow $d$ states are essential. Fig. 2 shows the band structure of $\mathrm{ZnS}$ calculated with $(+12) \mathrm{Zn} \mathrm{PP}$ and $(+20)$ Zn PP. We find that the LDA results do not show any significant difference on including the semicore states, implying that both PP's are good LDA PP's. For (+12) Zn PP, the top valence bands are almost identical in LDA and sX-LDA (after a rigid shift). The notable changes in sX-LDA are the band gap increase as well as the deepening of $d$ band position and the lower $\mathrm{Zn} s$ bands. Although improved by $\sim 0.7 \mathrm{eV}$ over LDA, the sX-LDA band gap, $2.41 \mathrm{eV}$, is too small compared with experimental value of $3.78 \mathrm{eV}$ whn $(+12) \mathrm{Zn} \mathrm{PP}$ is used. The inclusion of the whole Zn semicore states, as shown in Fig. 2 (b), further increase the gap between the valence bands and the conduction bands by $\sim 0.8 \mathrm{eV}$, and makes the comparison with experiment much better. The relative position of $d$ bands, however, undergoes a relatively small change, $\sim 0.2 \mathrm{eV}$.

The changes of band structure can be further analyzed by looking at the absolute eigenvalues of the KS equations. Fig. 3 shows the band edge states of $\mathrm{ZnS}$. The energies are plotted relative to the LDA valence band maximum (VBM) energy for each PP. The absolute eigenvalues in sX-LDA are generally downshifted compared with LDA eigenvalues. It is due to the fact that the screened-exchange potential is always negative. The sX-LDA band gaps are larger than those of LDA because the valence band are pull down even more than the conduction bands. The comparison between the sX-LDA resutls with different PP's shows that the inclusion of the semicore $s$ and $p$ states makes the conduction band downshift smaller, while keeping the valence bands and the $d$ states almost unchanged. In other words, the error in $d$ state wavefunctions in $(+12) \mathrm{Zn}$ PP causes the overestimation of the conduction band- $d$ states exchange interaction and leads to a underestimation of the band gap. 
In addition to ZnS, we studied effects of $d$ states in the sX-LDA for ZnSe, CdS, GaN, and InN. The results are summaries in Table II. For all the systems studied, the sX-LDA with $(+12 /+13)$ PP's, i.e., PP's without semicore $s$ and $p$ states, yields substantially smaller band gaps compared with experiments. When the full semicore states are included in PP's, the sX-LDA band gaps are improved and the results are comparable to GW results. The band gap discrepancy between the sX-LDA results and the experiements is less than $0.5 \mathrm{eV}$ in all cases. The $d$ electron binding energies (the energy differences between the VBM and the $d$ states) are improved from LDA. The $d$ electron binding energy discrepancy is also within $0.5 \mathrm{eV}$ from experimental values except GaN where a larger $1.3 \mathrm{eV}$ difference exists for its deep $(\sim-18 \mathrm{eV})$ d-level. In comparison, the $\mathrm{GW} d$ electron binding energy has larger error than sX-LDA.

We have also compared our plane-wave semicore PP sX-LDA results with all-electron sX-LDA results. The reported full potential linearized augmented plane-wave (FLAPW) sX-LDA band gaps from Ref. [8] are shown in Table II. The band gap difference between the PP and FLAPW sX-LDA are 0.31, 0.15, 0.09, and $0.43 \mathrm{eV}$ for CdS, ZnSe, GaN, and $\mathrm{ZnS}$, respectively. These differences are similar in amplitudes as the differences between the semicore PP and FLAPW LDA results. One problem is that due to our approximated way to generate the semicore PP, the LDA discrepancy between the semicore PP and FLAPW is slightly larger than difference between the conventional PP and FLAPW. Nevertheless, we believe the general good agreement between the semicore PP and FLAPW sX-LDA results comfirms the correctness of both methods.

\section{SUMMARY}

We have investigated the inclusion of d-electrons in the planewave pseudopotential screened-exchange LDA calculations. The inclusion of the cation d-electron is important in many II-VI and III-V semiconductors as its energy is close to the valence $\mathrm{s}$ and p levels. However, we found that when the conventional norm conserving LDA PP is used, the sX-LDA band gaps become much smaller than the experimental results. We have identified the problem: that is because the pseudo wavefunctions have deviated considerably from the all electron wavefunctions (especially for their center of mass positions). Although by construction, these pseudo wavefunctions can regenerate the all electron LDA results 
in bulk calculations, they cannot represent the screened exchange integrals of the all electron wavefunctions. We have thus generated PP with semicores. With the inclusion of the semicores, the pseudo wavefunctions become similar to the all electron wavefunctions, and the amplitudes of the screened exchange integrals have been restored. The same problem exists in planewave pseudopotential GW calculations, and similar semicore PP have been used previously for GW calculations. Our semicore PP sX-LDA band gaps agree well with the experimental ones, and the sX-LDA d-state energy levels agree better with experiment than the GW results. Our semicore PP sX-LDA results also agree well with the FLAPW sX-LDA results.

This work was supported by the DMS/BES/SC, and MICS/SC offices of the U.S. Department of Energy under Contract No. DE-AC02-05CH11231. It used the resources of National Energy Research Scientific Computing Center (NERSC). 
[1] P. Hohenberg and W. Kohn, Phys. Rev. 136, B864 (1964).

[2] W. Kohn and L. J. Sham, Phys. Rev. 140, A1133 (1965).

[3] J. C. Phillips, Phys. Rev. 112, 685 (1958).

[4] M. L. Cohen and V. Heine, The fitting of pseudopotentials to experimental data and their subsequent application (1970).

[5] M. T. Yin and M. L. Cohen, Phys. Rev. B 25, 7403 (1982).

[6] D. M. Bylander and L. Kleinman, Phys. Rev. B 41, 7868 (1990).

[7] A. Seidl, A. Gorling, P. Vogl, J. A. Majewski, and M. Levy, Phys. Rev. B 53, 3764 (1996).

[8] C. B. Geller, W. Wolf, S. Picozzi, A. Continenza, R. Asahi, W. Mannstadt, A. J. Freeman, and E. Wimmer, Appl. Phys. Lett. 79, 368 (2001).

[9] M. Moukara, M. Städele, J. A. Majewski, P. Vogl, and A.Görling, J. Phys. Conds. Matter 12, $6783(2000)$.

[10] F. R. Vukajlovic, E. L. Shirley, and R. M. Martin, Phys. Rev. B 43, 3994 (1991).

[11] M. Rohlfing, P. Krüger, and J. Pollmann, Phys. Rev. B 57, 6485 (1998).

[12] W. Luo, S. Ismail-Beigi, M. L. Cohen, and S. G. Louie, Phys. Rev. B 66, 195215 (2002).

[13] K. Lawniczak-Jablonska, T. Suski, I. Gorczyca, N. E. Christensen, K. E. Attenkofer, E. M. G. R. C. C. Perera, J. H. Underwood, D. L. Ederer, and Z. L. Weber, Phys. Rev. B 61, 16623 (2000).

[14] J. Wu, W. Walukiewicz, K. M. Yu, J. W. A. III, E. E. Haller, H. Lu, J. Schaff, Y. Saito, and Y. Nanishi, Appl. Phys. Lett. 80, 3967 (2002).

[15] A. Fleszar and W. Hanke, Phys. Rev. B 71, 045207 (2005).

[16] R. Weidemann, H.-E. Gumlich, M. Kupsch, and H.-U. Middelmann, Phys. Rev. B 45, 1172 (1992).

[17] L. Ley, R. A. Pollak, F. R. McFeely, S. P. Kowalczyk, and D. A. Shirley, Phys. Rev. B 9, 600 (1974).

[18] O. Madelung, M. Schulz, and H. Weiss, eds., Intrinsic Properties of Group IV Elements and III-V, II-VI, and I-VII Compounds, vol. 22 Pt. a of Landolt-Bornstein, New Series (Springer, Berlin, 1987).

[19] A. P. J. Stampfl, P. Hofmann, O. Schaff, and A. M. Bradshaw, Phys. Rev. B 55, 9679 (1997). 
[20] D. R. T. Zahn, G. Kudlek, U. Rossow, A. Hoffmann, I. Broser, and W. Richter, Adv. Mater. Opt. Electron. 3, 11 (1994).

[21] S. A. Ding, G. Neuhold, J. H. Weaver, P. Häberle, K. Horn, O. Brandt, H. Yang, and K. Ploog, J. Vac. Sci. Technol. A 14, 819 (1996). 
TABLE I: Comparison of atomic levels in all-electron and pseudopotential LDA results. $\epsilon_{l}^{P P}$, and $\epsilon_{l}^{A E}$ are the highest occupied atomic eigenvalue with $l$ angular momentum from $(+20 /+21)$ pseudopotential and all-electron calculations, respectively. $\left.K_{l l^{\prime}}(+12 /+13), K_{l l^{\prime}}(+20 /+21)\right)$, and $K_{l l^{\prime}}(A E)$ are the screened-exchange integrals evaluated using $\phi_{l}(r)$, from $(+12 /+13)$ pseudopotential, $(+20 /+21)$ pseudopotential, and all-electron wavefunctions, respectively. We used the same screening length as in the test bulk calculations; $\mathrm{ZnS}, \mathrm{CdS}, \mathrm{GaN}$, and $\mathrm{InN}$ valence electron average densities were used for $k_{T F}$ in $\mathrm{Zn}, \mathrm{Cd}$, Ga, and $\mathrm{In}$ atoms, respectively. We averaged the screened-exchange integral for an angular momentum over the magnetic quantum numbers.

\begin{tabular}{c|cccc}
\hline \hline & $\mathrm{Zn}$ & $\mathrm{Cd}$ & $\mathrm{Ga}$ & $\mathrm{In}$ \\
\hline$\epsilon_{s}^{P P}-\tilde{\epsilon}_{s}^{A E}(\mathrm{eV})$ & -0.034 & -0.052 & -0.029 & -0.073 \\
$\epsilon_{p}^{P P}-\tilde{\epsilon}_{p}^{A E}(\mathrm{eV})$ & & & -0.004 & 0.007 \\
$\epsilon_{d}^{P P}-\tilde{\epsilon}_{d}^{A E}(\mathrm{eV})$ & 0.089 & 0.112 & 0.144 & 0.163 \\
\hline$K_{s s}(+12 /+13) / K_{s s}(A E)$ & 1.04 & 1.11 & 1.04 & 1.10 \\
$K_{s s}(+20 /+21) / K_{s s}(A E)$ & 1.05 & 1.13 & 1.05 & 1.13 \\
\hline$K_{s p}(+12 /+13) / K_{s p}(A E)$ & 1.02 & 1.05 & 1.03 & 1.05 \\
$K_{s p}(+20 /+21) / K_{s p}(A E)$ & 1.01 & 1.03 & 1.02 & 1.03 \\
\hline$K_{s d}(+12 /+13) / K_{s d}(A E)$ & 2.38 & 1.85 & 1.91 & 2.10 \\
$K_{s d}(+20 /+21) / K_{s d}(A E)$ & 1.15 & 1.24 & 1.16 & 1.24 \\
\hline$K_{p p}(+12 /+13) / K_{p p}(A E)$ & 1.01 & 1.02 & 1.01 & 1.02 \\
$K_{p p}(+20 /+21) / K_{p p}(A E)$ & 1.00 & 1.02 & 1.01 & 1.01 \\
\hline$K_{p d}(+12 /+13) / K_{p d}(A E)$ & 1.99 & 1.60 & 1.50 & 1.72 \\
$K_{p d}(+20 /+21) / K_{p d}(A E)$ & 1.10 & 1.13 & 1.14 & 1.12 \\
\hline$K_{d d}(+12 /+13) / K_{d d}(A E)$ & 0.62 & 0.94 & 0.79 & 0.90 \\
$K_{d d}(+20 /+21) / K_{d d}(A E)$ & 0.99 & 0.99 & 0.98 & 0.99 \\
\hline \hline
\end{tabular}


TABLE II: The band gap energy $\left(E_{g}\right)$ and the semicore $d$ state binding energy $\left(E_{d}\right)$ in eV. All systems were calculated for the zinc-blende structure using the experimental lattice constants. The $d$ state binding energy is defined as the eigenvalue difference between the top most valence band state energy and the highest $d$ state energy at $\Gamma$ point. $\mathrm{PP}$ and $\mathrm{AE}$ denote pseudopotential and FLAPW calculations, respectively. For PP LDA and PP sX-LDA results, the numbers outside and inside the parenthesis are the results using $(+20 /+21)$ PP's and $(+12 /+13)$ PP's, respectively, For comparability reason, we list planewave LDA based GW calculation results with a random-phase approximation for the screened Coulomb interaction and a plasmon-pole model for the dynamics of the screening. In all these calculations, the spin-orbit effects were not taken into account.

\begin{tabular}{lc|ccccc}
\hline \hline & & ZnSe & ZnS & CdS & GaN & InN \\
\hline PP LDA & $E_{g}$ & $0.94(0.97)$ & $1.66(1.70)$ & $0.84(0.84)$ & $1.87(1.69)$ & $-0.38(-0.42)$ \\
& $E_{d}$ & $-6.21(-6.61)$ & $-5.91(-6.22)$ & $-7.17(-7.46)$ & $-12.58(-13.65)$ & $-12.79(-13.31)$ \\
\hline PP sX-LDA & $E_{g}$ & $2.42(1.41)$ & $3.24(2.41)$ & $2.06(1.78)$ & $2.95(2.40)$ & $0.39(0.08)$ \\
& $E_{d}$ & $-9.59(-9.32)$ & $-8.83(-8.64)$ & $-8.71(-9.23)$ & $-16.39(-17.35)$ & $-14.61(-15.26)$ \\
\hline AE LDA & $E_{g}$ & 1.03 & 1.81 & 0.82 & 1.59 & -0.49 \\
\hline AE sX-LDA & $E_{g}$ & $2.57^{a}$ & $3.67^{a}$ & $2.37^{a}$ & $3.04^{a}$ & \\
\hline GW & $E_{g}$ & $2.32^{b}, 2.24^{c}$ & $3.19^{b}, 3.50^{d}, 3.38^{c}$ & $2.45^{d}, 2.11^{c}$ & $2.88^{d}$ & \\
\hline Experiment & $E_{g}$ & $2.82^{e}$ & $3.78^{e}$ & $2.48^{f}$ & $3.3^{g}$ & $0.8^{h}$ \\
& $E_{d}$ & $-9.37^{i},-9.20^{j}$ & $-9.0^{e}$ & $-9.2^{k}$ & $-17.7^{l}$ & \\
\hline \hline
\end{tabular}

${ }^{a}$ Reference 8.

${ }^{g}$ Reference 13.

${ }^{b}$ Reference 12 .

${ }^{h}$ Reference 14.

${ }^{c}$ Reference 15.

${ }^{i}$ Reference 16.

${ }^{d}$ Reference 11.

${ }^{j}$ Reference 17.

${ }^{e}$ Reference 18.

${ }^{k}$ Reference 19.

${ }^{f}$ Reference 20.

${ }^{l}$ Reference 21 . 


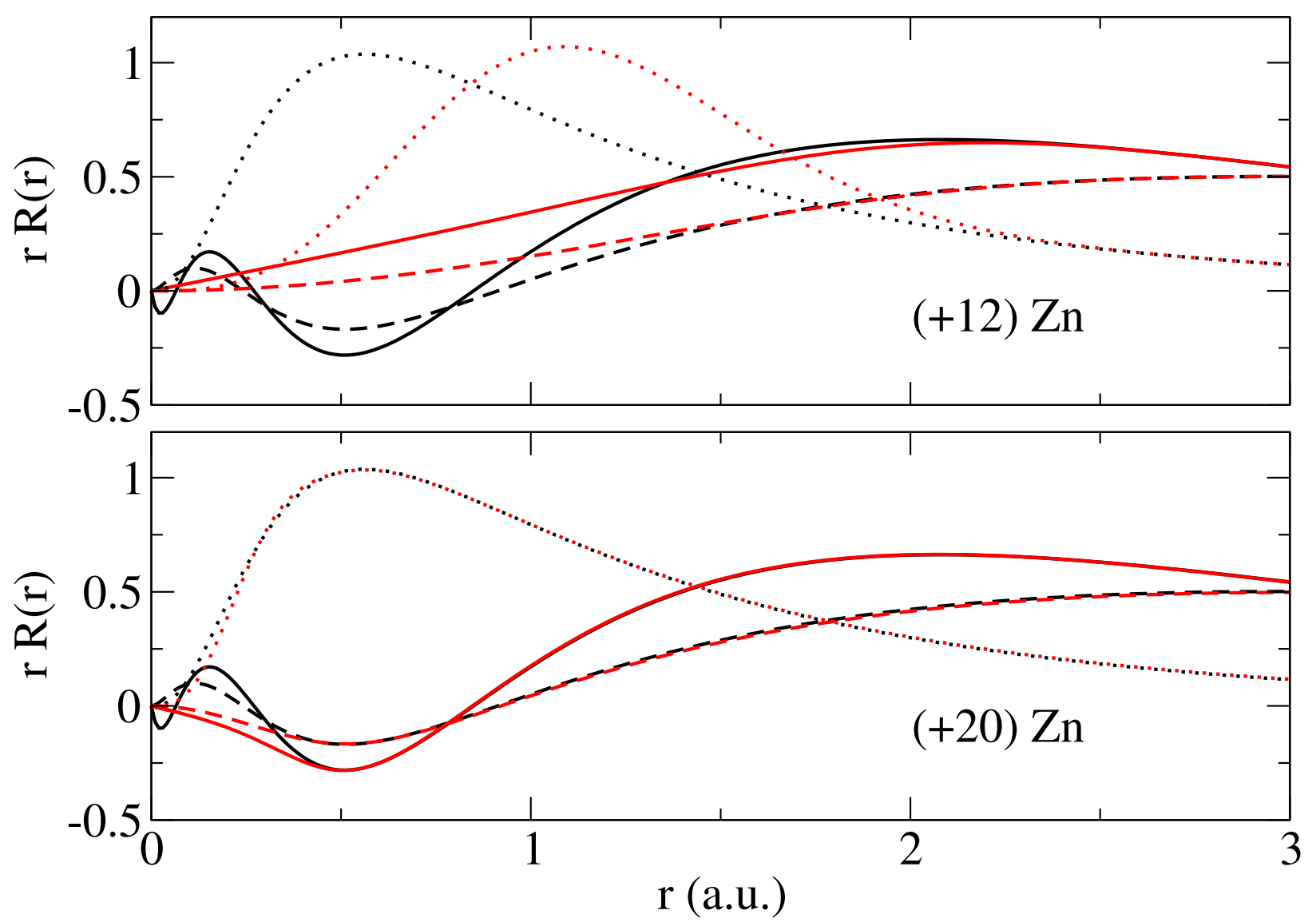

FIG. 1: Radial wavefunctions, $u(r)=r R(r)$, of Zn atom calculated in LDA. Upper panel: Allelectron wavefunctions (black lines) and $(+12)$ Zn pseudopotential wavefunctions (red lines) are shown. Solid, dashed, and dotted lines are for $s, p$, and $d$ angular momentum, respectively. Lower panel: Same as the upper panel except that $(+20) \mathrm{Zn}$ pseudopotential is used instead of $(+12) \mathrm{Zn}$. 

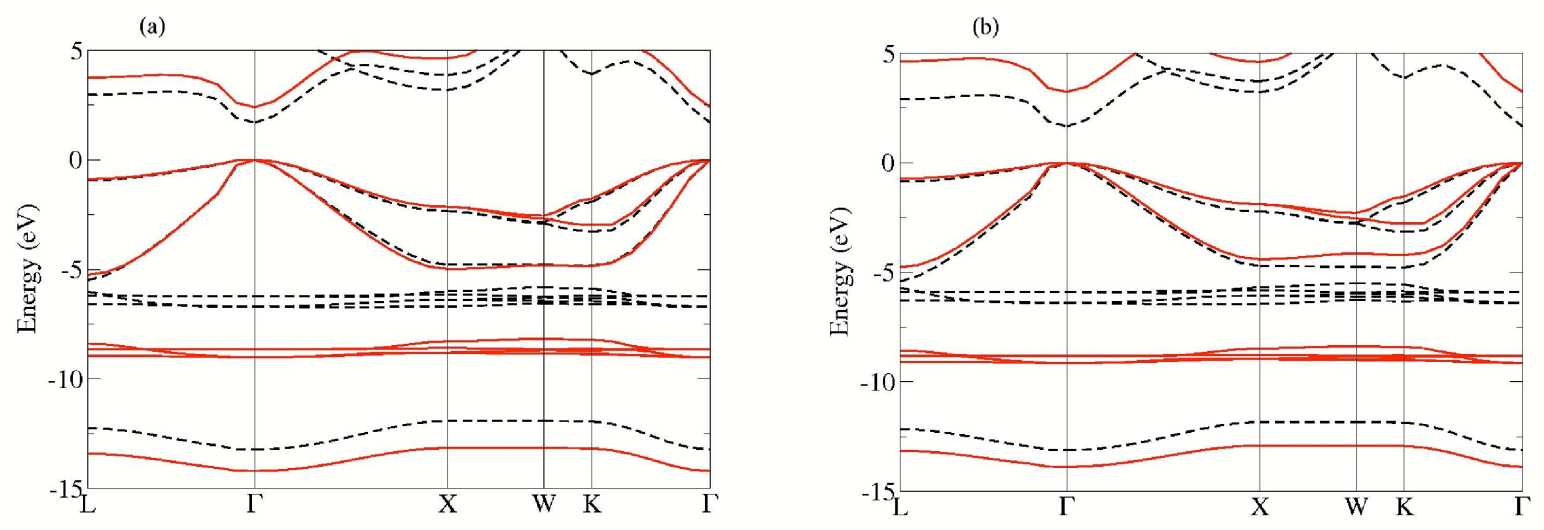

FIG. 2: Band structure of zinc-blende ZnS. The dashed and solid lines denote the electron bands calculated from LDA and sX-LDA, respectively. (a) $\mathrm{Zn}^{+12}$ core is used for the $\mathrm{Zn}$ pseudopotential. (b) $\mathrm{Zn}^{+20}$ core is used for the $\mathrm{Zn}$ pseudopotential. LDA and sX-LDA bands are shifted so that the valence band maximum be placed at zero energy. 


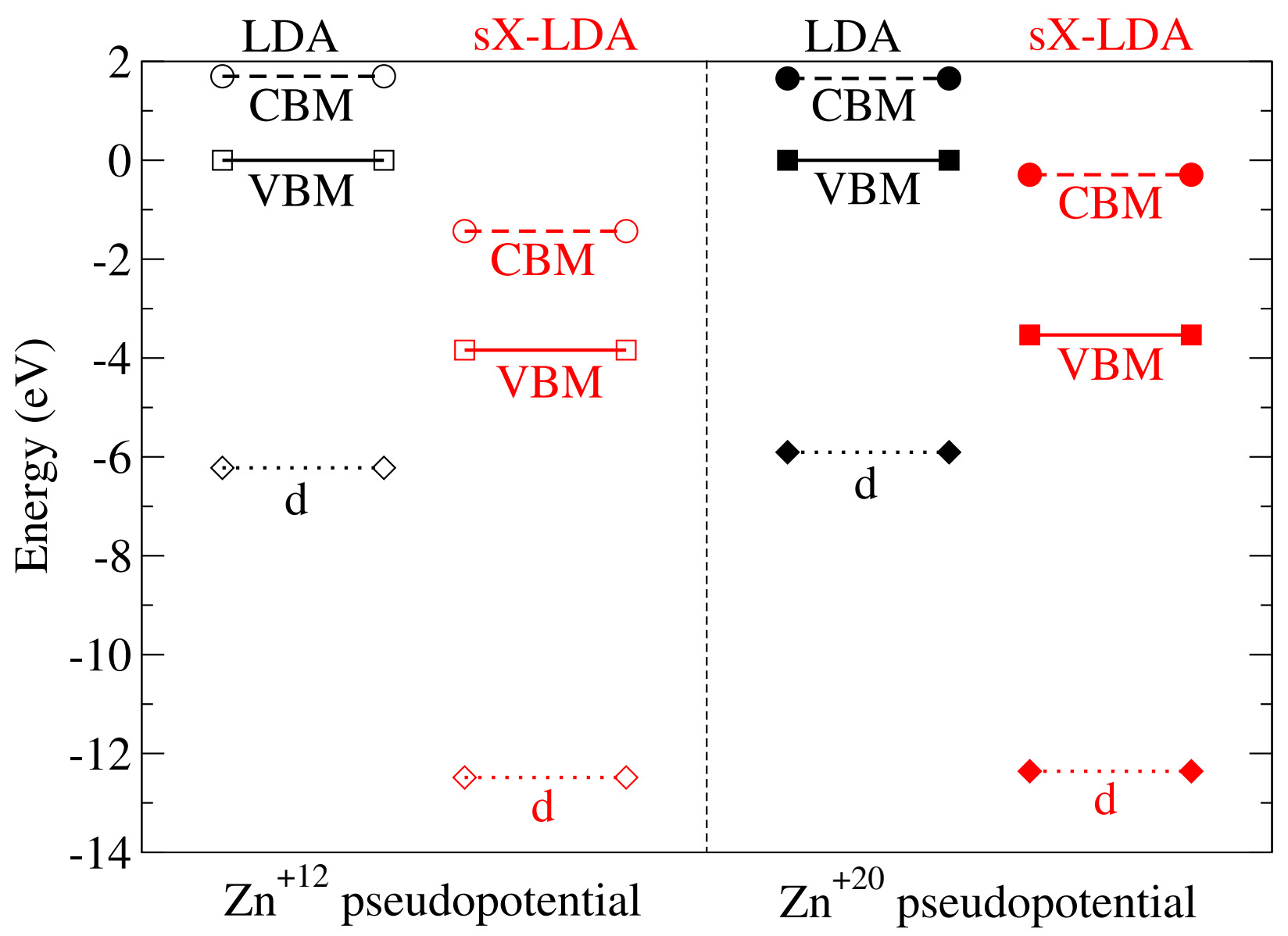

FIG. 3: Band edge states of zinc-blende ZnS. The dashed, solid, and dotted lines denote the electron energy at CBM, VBM, and top $d$ band state in order. Black and red lines correspond to LDA and sX-LDA, respectively. LDA and sX-LDA bands are shifted relative to the LDA VBM energy. 